\title{
APLICACIÓN DE ESPECTROMETRÍA DE RAYOS X PARA EL ESTUDIO DE LA MADERA: ALGUNOS RESULTADOS INICIALES.
}

\section{X-RAYS SPECTROMETRIC METHODS FOR WOOD PROPERTIES DETERMINATION: SOME INITIAL RESULTS}

\author{
Ricardo Baettig ${ }^{1}$,Patrick Perré ${ }^{2}$, Romain Rémond
}

\section{RESUMEN}

El objetivo de este artículo es mostrar la posibilidad de utilizar fuentes policromáticas de rayos X en experimentos relacionados con la madera en que normalmente se requiere de fuentes monocromáticas. Se presentan diferentes aplicaciones de espectrometría de rayos $\mathrm{X}$ en base a fuentes policromáticas conformadas por tubos sin dispositivos de monocromatización, pero usando detectores espectrométricos de alta resolución energética. En primer lugar se describe una aplicación de transmisión o atenuación de rayos $\mathrm{X}$ destinada a obtener la variación espacio-temporal del contenido de humedad al interior de una tabla en proceso de secado a $70^{\circ} \mathrm{C}$ y $50^{\circ} \mathrm{C}$ en bulbo seco y bulbo húmedo respectivamente. Esta aplicación permitió realizar una cartografía digital del interior de la tabla y se reveló útil para caracterizar el proceso de evacuación de la humedad desde el interior de la madera. Luego se presenta un equipamiento innovador diseñado y fabricado por los autores para registrar la intensidad de dispersión coherente de rayos X. Los resultados demuestran la posibilidad de acceder al contenido de humedad y el ángulo microfibrilar, cuantificando las señales espectrales registradas tanto para el agua como para el plano cristalino (002) de la celulosa. La existencia de una señal de fondo originada por la fase amorfa de la madera impidió realizar estimaciones cuantitativas de contenido de humedad cuando la densidad anhidra de la madera es desconocida.

Palabras clave: Rayos X, contenido de humedad, dispersión coherente, ángulo microfibrilar

\begin{abstract}
We investigated the possibilities to use polychromatic X-rays sources to study wood properties that usually require of monochromatic radiation. Three applications using $\mathrm{X}$-ray tubes without monochromator together with high-resolution energy-sensitive X-rays detectors are presented. In a first experiment, a standard X-ray source and a spectrometric detector were placed on both sides of a wood dryer which operates at $70^{\circ} \mathrm{C}$ and $50^{\circ} \mathrm{C}$ for dry-bulb and wet-bulb respectively. Digital cartography obtained can offer the possibility to identify some phenomenological parameters of fluid migration in wood. In a second part, an innovative device designed and made by the authors to record X-ray scatter intensities is presented. The feasibility to use the ratio of diffraction to attenuation for moisture content determination is discussed. This device has been also used for microfibril angle determination by recording and analyzing the $X$-ray scattered intensities of cellulose (002) lattice. The microfibril angle determination was successful; however, a background of X-rays scattering from amorphous component of wood prevented the determination of moisture content.
\end{abstract}

Keywords: X-ray absorptiometry, moisture content, X-ray scattering, microfibril angle

${ }^{1}$ Centro Tecnológico del Álamo. Facultad de Ciencias Forestales. Universidad de Talca. Talca. Chile. rbaettig@utalca.cl

${ }^{2}$ LERMAB (Laboratoire d'Etudes et de Recherche sur le Matériau Bois) - UMR 1093 INRA ENGREF.14, rue Girardet. Nancy, 54042, France. perre@lermab-engref.fr

Autor para correspondencia: rbaettig@utalca.cl

Recibido: 26.04. 2007. Aceptado: 29.10.2007 


\section{INTRODUCCIÓN}

Los rayos X se producen comúnmente mediante el bombardeo con electrones de un blanco metálico dentro de un tubo al vacío. Cuando los electrones impactan en el ánodo la casi totalidad de la energía cinética es disipada como calor, pero una pequeña proporción genera fotones altamente energéticos o rayos $\mathrm{X}$. La distribución energética de los fotones generados por frenado resultada ser continua, sin embargo, también se producen unas líneas espectrales características del metal que compone el ánodo. Estas líneas corresponden a transiciones o saltos de energía de los electrones dentro de las capas atómicas. Para identificar estas transiciones se utiliza la notación de Siegbahn. Por ejemplo, cuando se produce un espacio en la capa $\mathrm{K}$ que es rellenado por un electrón situado 2 capas más arriba se habla de una transición $K \beta$. De este modo, eligiendo convenientemente el elemento químico que compone el ánodo es posible obtener un fuerte flujo de fotones $\mathrm{X}$ a una energía específica, aún cuando el continuo de fondo o "Bremsstrahlung" siga presente llegando hasta una energía máxima equivalente al voltaje aplicado al tubo. En cuanto a la detección, la mayor parte de las aplicaciones de radiaciones ionizantes utilizan detectores de centelleo debido a que estos permiten realizar una cartografía digital bidimensional de los materiales estudiados. Sin embargo, esta tecnología no permite una adecuada discriminación energética o "espectrometría" de la radiación cuando se usan rayos policromáticos. Por otra parte, dada su baja sensibilidad, requieren de una relativa alta intensidad de radiación. Al contrario, la utilización de transductores de rayos $\mathrm{X}$ en base a diodos de estado sólido ha simplificado la obtención de análisis espectrométricos debido a que estos dispositivos son sensibles a la energía portada por los fotones que los impactan. La utilización de materiales tales como el Teluro de Cadmio ha permitido eliminar la necesidad de enfriamiento criogénico reemplazándola por microdispositivos basados en el efecto termoeléctrico (Peltier). De este modo los detectores han pasado a ser portátiles y de bajo costo relativo.

El análisis espectral, permite entre otras cosas, realizar una discriminación energética de la radiación abriéndose la posibilidad de utilizar un simple tubo de rayos $\mathrm{X}$ en aplicaciones en las que tradicionalmente se han utilizado radiaciones monocromáticas provenientes ya sea desde radioisótopos (rayos $\gamma$ ), aceleradores de partículas o desde cristales monocromatizadores adosados a tubos de rayos $\mathrm{X}$ de alta potencia.

Cuando un rayo X penetra en un medio se constata una disminución progresiva de su flujo que se debe a la interacción de los fotones con los electrones internos de los átomos que lo constituyen. La energía extraída del rayo incidente se encuentra bajo dos formas: una parte es absorbida por el material y otra parte es difundida o desviada de la dirección del rayo incidente. La disminución de flujo de fotones se rige por la conocida ley exponencial de Beer-Lambert, donde el parámetro $\mu(E)$ o coeficiente de atenuación másico de los rayos X se describe por una curva característica para cada elemento químico en función de la energía fotónica del rayo incidente. La detección de distintos tipos de interacción entre fotones y los átomos permite inferir acerca de la composición química y la estructura del material.

El análisis espectral de los rayos X re-emitidos por fluorescencia permite la obtención de "firmas" características de los distintos elementos químicos presentes en la muestra. Análisis de las intensidades relativas de cada firma permiten obtener concentraciones de elementos. En ciencias de la madera la fluorescencia de rayos X se utiliza para la medición de la retención de preservantes en madera tratada o para el estudio fisiológico de la concentración de macro y microelementos en el tejido leñoso. Por otra parte, si el material está constituido por dos componentes suficientemente distintos químicamente, el análisis de la curva que resulta de la mezcla de ambos puede servir para determinar la proporción de la mezcla. Estos análisis multienergéticos (dual energy X-ray absorptiometry - DEXA) se han utilizado tradicionalmente en el campo médico para la estimación de la densidad de huesos rodeados por los tejidos circundantes (Lasquey y Phil 1996, Dinten et al. 2001). Se ha planteado el mismo principio 
para la discriminación cuantitativa de otras parejas de materiales tales como: suelo-petróleo (Norel, 1965, Clarijs et al. 1999), suelo-agua (Oostrom y Dave 1990, McBride y Miller 1994, Bauters et al. 2000, Schena et al. 2002), vidrio-plástico (Letang et al. 2003), concreto-agua (Hansen et al. 1999, Bentz y Hansen 2000, Hu y Stroeven 2003) o madera-agua (Perré y Thiercelin 1997, 2004, Baettig et al. 2006). Así mismo, el estudio de la dispersión coherente de rayos $\mathrm{X}$ que se debe a la interferencia cooperativa de los fotones se usa corrientemente para el estudio de la estructura cristalina de materiales. En madera, la difracción de rayos X es utilizada para determinar el ángulo microfibrilar casi siempre basándose en la difracción provocada por el plano atómico (002) del cristalito de celulosa, el cual, se orienta paralelo a la microfibrila. Dado que el espaciamiento interatómico de este plano alcanza 0,39 $\mathrm{nm}$, según la ecuación de Bragg la radiación de Cobre se difracta de un ángulo igual a $22^{\circ}$ respecto del rayo transmitido a través de la muestra. El análisis de la variación azimutal de la intensidad de difracción permite realizar estimaciones del ángulo microfibrilar mediante relaciones empíricas (Kretschmann et al. 1997, Evans et al. 1996, Evans 1999, Verrill et al. 2001). De modo parecido a un material cristalino, el agua líquida genera dispersión coherente de rayos $\mathrm{X}$ aunque con una intensidad extremadamente débil. Este fenómeno es intensamente estudiado en el campo médico (Johns et al. 2002, Tartari et al. 2002, Poletti et al. 2004), para la detección de sustancias prohibidas y para la evaluación de proporciones en mezclas agua/hidrocarburos en la industria petroquímica (Key, 1999). En el campo de la madera, se han realizado estudios para la determinación del contenido de humedad por medio de la discriminación energética de las señales difractadas por la fase cristalina de la madera y por el agua en muestras de madera húmeda (Baettig y Perré, 2006).

El objetivo de este artículo es probar la factibilidad de utilizar fuentes policromáticas de rayos X en experimentos relacionados con la madera en que normalmente se requiere de fuentes de rayos $\mathrm{X}$ monocromáticas. Se presentan dos aplicaciones concretas para el análisis cuantitativo de variaciones espacio-temporales del contenido de humedad de la madera en proceso de secado y una aplicación a la estimación del ángulo microfibrilar (AMF) de la madera sólida seca. En primer lugar se describe un experimento de cartografía digital de rayos $\mathrm{X}$ de una tabla verde en proceso de secado convectivo. Se intenta determinar la evolución espacio-temporal del contenido de humedad en el espesor de la tabla, a lo largo de todo el proceso de secado. El propósito final de este tipo de experimentos es llegar a determinar parámetros del secado que son necesarios para alimentar simuladores cuantitativos de secado tal como TransPore (Perré y Turner 1996, 1999).

Dado que la variación espacial de la humedad se superpone con la variación de densidad de la madera, la determinación del contenido de humedad se realizó una vez conocida la variación de densidad de la tabla, determinada en un experimento separado, realizado al estado anhidro. Para solucionar esta dificultad e intentar determinar el contenido de humedad en tiempo real durante el secado de la madera se realizó un segundo experimento. Este consistió en registrar las modificaciones a la "firma" en dispersión coherente de rayos X dejada por las moléculas de agua contenidas en una muestra secándose. Para ello se construyó un sistema especial capaz de registrar simultáneamente las señales de atenuación y dispersión de rayos X y el peso de la muestra.

Finalmente, este mismo dispositivo se utilizó para registrar experimentalmente la firma en difracción de rayos $\mathrm{X}$ dejada por la madera seca, con el propósito de alimentar un modelo físico-geométrico para estimar el ángulo microfibrilar de las muestras. El propósito de este modelo matemático es el de contribuir a determinar de una manera más precisa esta propiedad de la madera, por medio de la toma en consideración de la geometría real de la pared celular. 


\section{MATERIAL Y MÉTODO}

Se utilizaron 2 equipos de rayos $\mathrm{X}$ diseñados por el equipo de investigación del Laboratorio de Ciencias de la Madera (LERMAB) en Nancy-Francia entre 2002 y 2004. El primer equipo consistió de un tubo de rayos X marca Rontgen-Technik con ánodo en tungsteno. Este equipamiento se adosó en la parte exterior de un costado de un cilindro de secado multipropósito capaz de realizar secado convectivo o por alta frecuencia en presiones de hasta 0,1 bares y temperaturas de hasta $150^{\circ} \mathrm{C}$. En la parte externa del otro extremo de la cámara se secado se dispuso un detector espectrométrico de rayos X. Tanto la fuente como el detector fueron acoplados a unos sistemas de desplazamiento a comando numérico que permitieron realizar el barrido en el espesor de una pieza de madera de tamaño real (Fig. 1).

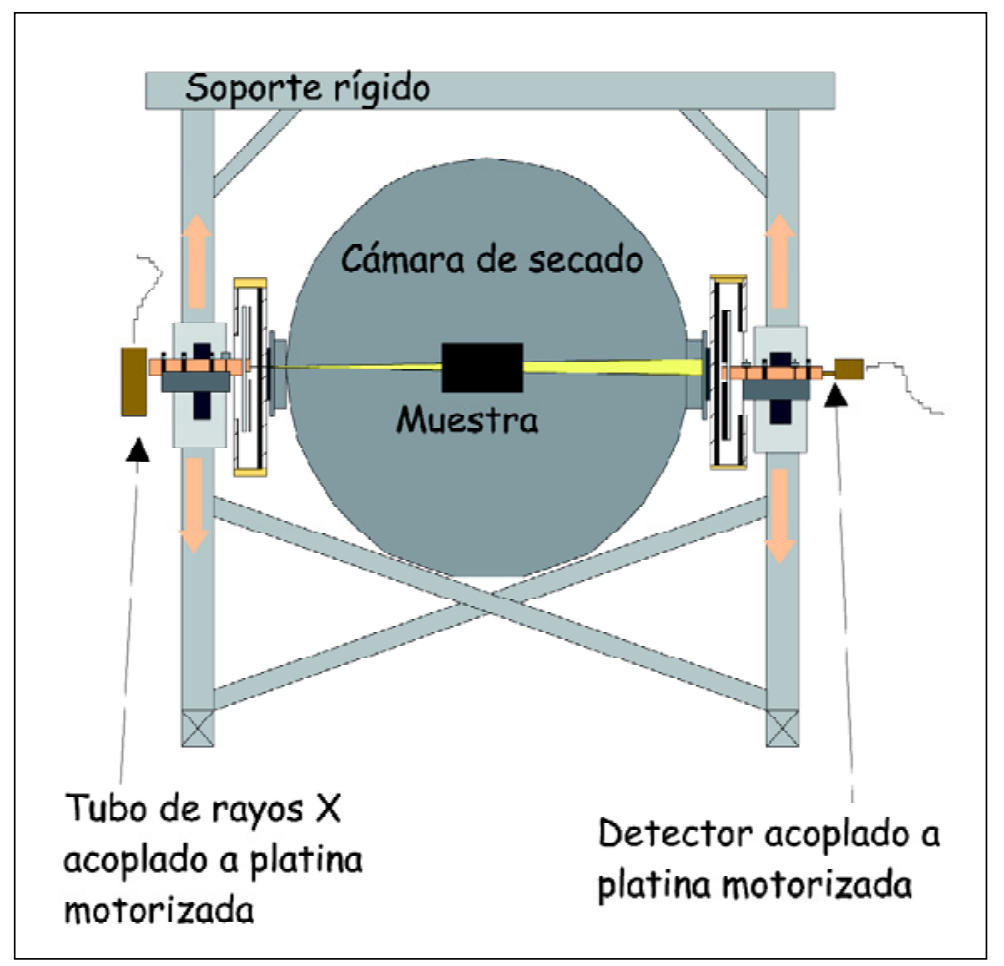

Figura 1. Equipamiento para determinación de perfiles de humedad durante el secado.

El segundo equipo consistió de una caja blindada por láminas de plomo al cual se adosó una fuente de cristalografía de rayos X. Dentro de la camisa de protección se dispusieron alternativamente dos modelos de tubos de rayos X: uno con ánodo de cobre marca Philips y otro con ánodo de molibdeno marca AEG. La fuente de alimentación consistió de un generador marca INEL modelo XRG 3000. Una tubería de latón cargado en plomo con paredes de $15 \mathrm{~mm}$ de espesor y casi $1 \mathrm{~m}$ de longitud sirvió para guiar los rayos X entre la fuente y la caja blindada, al interior de la cual se dispusieron las muestras de madera y los detectores (Fig. 2). Los detectores espectrométricos utilizados correspondieron al modelo XR-100T del fabricante Amptek. Este consiste en un detector puntual de CdTe o CdZnTe con un tamaño del elemento sensible de alrededor de $9 \mathrm{~mm}^{2}$. La adquisición y tratamiento de señales fue realizado por medio de amplificadores modelo PX2T de Amptek y por analizadores multicanal modelo MCA8000A del mismo fabricante. La resolución energética fue de alrededor de $0,4 \mathrm{keV}$ y se utilizaron las líneas de emisión $K \alpha$ y $K \beta$ del cobre $(8,05-8,90 \mathrm{keV})$ y molibdeno $(17,48-19,61 \mathrm{keV})$ junto a la fluorescencia del indio $(24,21-27,27 \mathrm{keV})$ para calibrar los analizadores multicanal. 


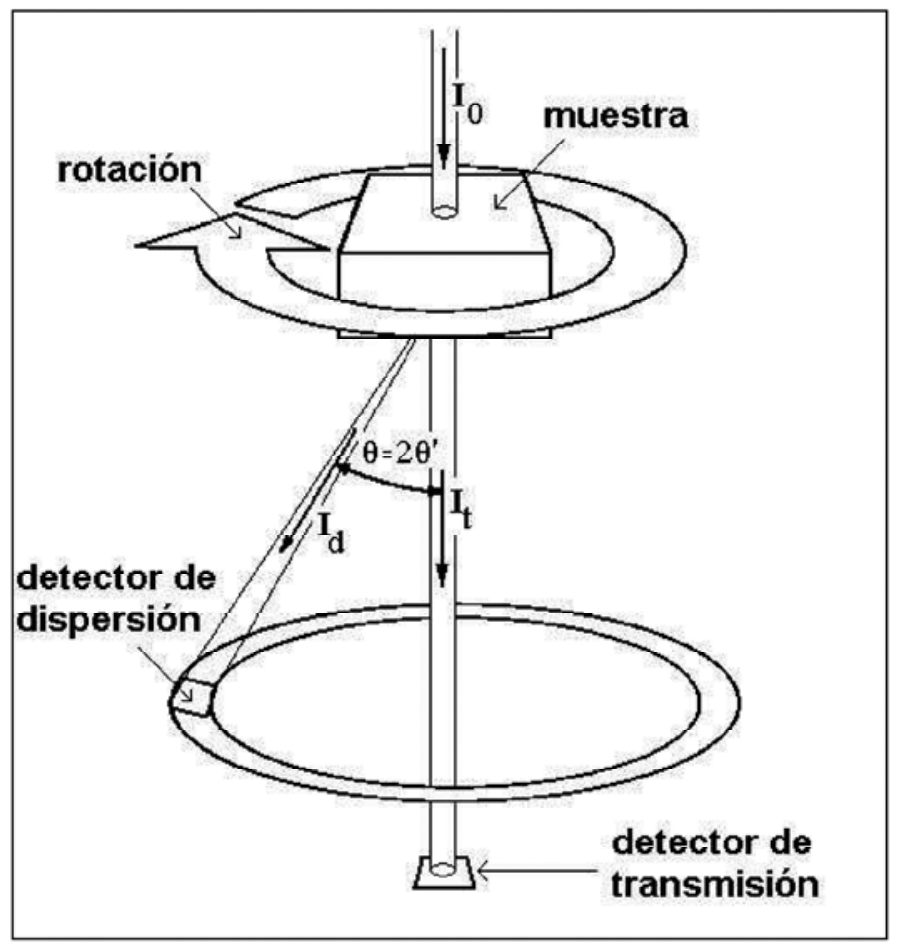

Figura 2. Esquema de ensayo de difracción de rayos X.

Métodos de atenuación de rayos X: se realizó un experimento determinación de la evolución espaciotemporal del contenido de humedad de la madera mediante un experimento de transmisión de rayos $\mathrm{X}$ en la cámara de secado multipropósito del LERMAB. Se realizaron 2 ensayos independientes en tablas de 28 y $65 \mathrm{~mm}$ de espesor usando un barrido con resolución espacial de alrededor de $5 \mathrm{~mm}$ demorando 30 minutos o una hora en cada caso para completar el escaneo completo del espesor. Se utilizó la especie Picea abies (L.) Karst en estado verde a condiciones de temperatura de bulbo seco y bulbo húmedo constantes de $70^{\circ} \mathrm{C}$ y $50^{\circ} \mathrm{C}$ respectivamente, para obtener un contenido de humedad final cercano a 10\%. La duración total de cada experimento fue de 3 y 5 días en las piezas de menor y mayor espesor respectivamente. La determinación del contenido de humedad a cada instante del secado se realizó a posteriori por medio de un análisis de la curva de coeficientes de atenuación $\mu(E)$ (absorciometría de rayos X) en un rango de energía de 12 a $30 \mathrm{keV}$ (Baettig et al. 2006). Como resultado se presenta la evolución temporal de los perfiles de humedad en el espesor de las tablas.

Métodos de dispersión coherente de rayos X: se realizaron dos experimentos usando pequeñas muestras de madera colocadas al interior de caja blindada descrita previamente. Un primer tipo de experimento consistió en registrar la evolución de la intensidad de la dispersión coherente de rayos $\mathrm{X}$ provocada por una muestra húmeda sometida a un secado a temperatura ambiente. Un segundo tipo de experimento consistió en realizar ensayos de determinación de la intensidad de la dispersión coherente generada por el plano (002) en madera seca. Ahora bien, debido a la configuración puntual de los detectores, para lograr la obtención de un perfil de difracción alrededor de un arco de $360^{\circ}$ se tuvo que hacer rotar la muestra en un eje imaginario coincidiendo con el rayo incidente. Esto se realizó por medio de un mecanismo fabricado especialmente por los autores, en base a dispositivos de control numérico marca Newport-Microcontrole. Por otra parte, otro mecanismo de desplazamiento lineal del mismo fabricante permitió realizar translaciones en la dirección vertical, con el propósito de apoyar periódicamente la muestra en una balanza electrónica. Este sistema permitió realizar mediciones automáticas de la pérdida de peso de la muestra de madera sin necesidad de extraerla ni de tocarla. 
En el caso de madera húmeda, se utilizó una muestra verde de Picea abies (L.) Karst. Se estudió la evolución del cuociente $R^{\prime}=I_{d}(E) / I_{t}(E)$ descrito inicialmente por Magalhaes et al. (1995). Este es el cuociente calculado para cada nivel de energía, entre la intensidad de difracción y la intensidad de transmisión a distintos contenidos de humedad. Las condiciones de secado fueron las condiciones ambientales de sala, las que se mantuvieron alrededor de $24^{\circ} \mathrm{C}$ y $40 \%$ de humedad relativa durante todo el experimento. Al cabo de 48 horas de registro la muestra fue extraída, llevada al estado anhidro y vuelta a ubicar en el dispositivo de rayos X. Dado que no fue alterada la potencia de emisión del generador, se pudo volver a registrar el cuociente $I_{d}(E) / I_{t}(E)$ en la misma muestra, pero al estado anhidro. Operacionalmente la muestra fue hecha rotar en pasos angulares (azimutales) de $15^{\circ}$ y en cada una de estas posiciones se registraron simultáneamente los espectros de transmisión $\left(\mathrm{a} 0^{\circ}\right)$ y de dispersión (a $10,5^{\circ}$ ) por medio de la utilización de ambos detectores. Tomando en cuenta las pérdidas de tiempo generadas por la pesada de la muestra realizada cada 5 minutos, la obtención de un perfil completo abarcando los $360^{\circ}$ demoró alrededor de una hora (la duración de la adquisición de espectros fue de 60 segundos en cada posición azimutal). Durante las 48 horas se completaron varios giros completos de la muestra lo que permitió obtener una varianza angular para cada nivel de energía fotónica.

En el caso se madera seca se confrontaron perfiles de difracción de la línea de emisión $K \alpha$ del molibdeno medidos experimentalmente en muestras de madera temprana, madera tardía y madera de compresión de Picea abies (L.) Karst contra perfiles teóricos obtenidos mediante una simulación geométrica que toma en cuenta la geometría de las paredes celulares. Cada muestra de $5 \mathrm{~mm}$ de espesor fue hecha rotar en pasos angulares de alrededor de $2^{\circ}$ y el detector de posicionó a un ángulo $2 \theta$ de $10,5^{\circ}$. El tiempo de adquisición de los espectros en cada posición fue de $60 \mathrm{~s}$, demorando alrededor de 3 horas la obtención de un perfil completo, es decir, abarcando $360^{\circ}$. La simulación de la intensidad de difracción se realizó en base a un modelo físico-geométrico desarrollado por los autores y alimentado por la distribución de la orientación de las paredes celulares. Detalles acerca de la concepción de este modelo se encuentra en (Baettig, 2005). La distribución de la orientación de las paredes celulares se obtuvo mediante tratamiento de imágenes digitales. Dichas imágenes fueron obtenidas desde las mismas muestras usando un microscopio electrónico marca FEI modelo Quanta 200.

\section{RESULTADOS}

La figura 3 presenta la evolución temporal del perfil de humedad de la tabla de $28 \mathrm{~mm}$ de espesor sometida a secado convectivo. En una etapa inicial, el perfil es relativamente plano revelando la homogeneidad inicial del contenido de humedad de la madera. Posteriormente se aprecia que la humedad del borde es muy inferior al del centro de la tabla (los perfiles adoptan una forma aproximadamente parabólica). Incluso a partir de 12 horas de iniciado el proceso, la parte externa de la tabla entra en la zona higroscópica (bajo 28-30\% de contenido de humedad). Posteriormente el perfil va descendiendo mucho más lentamente para llegar a hacerse casi plano pasadas 72 horas de secado. 


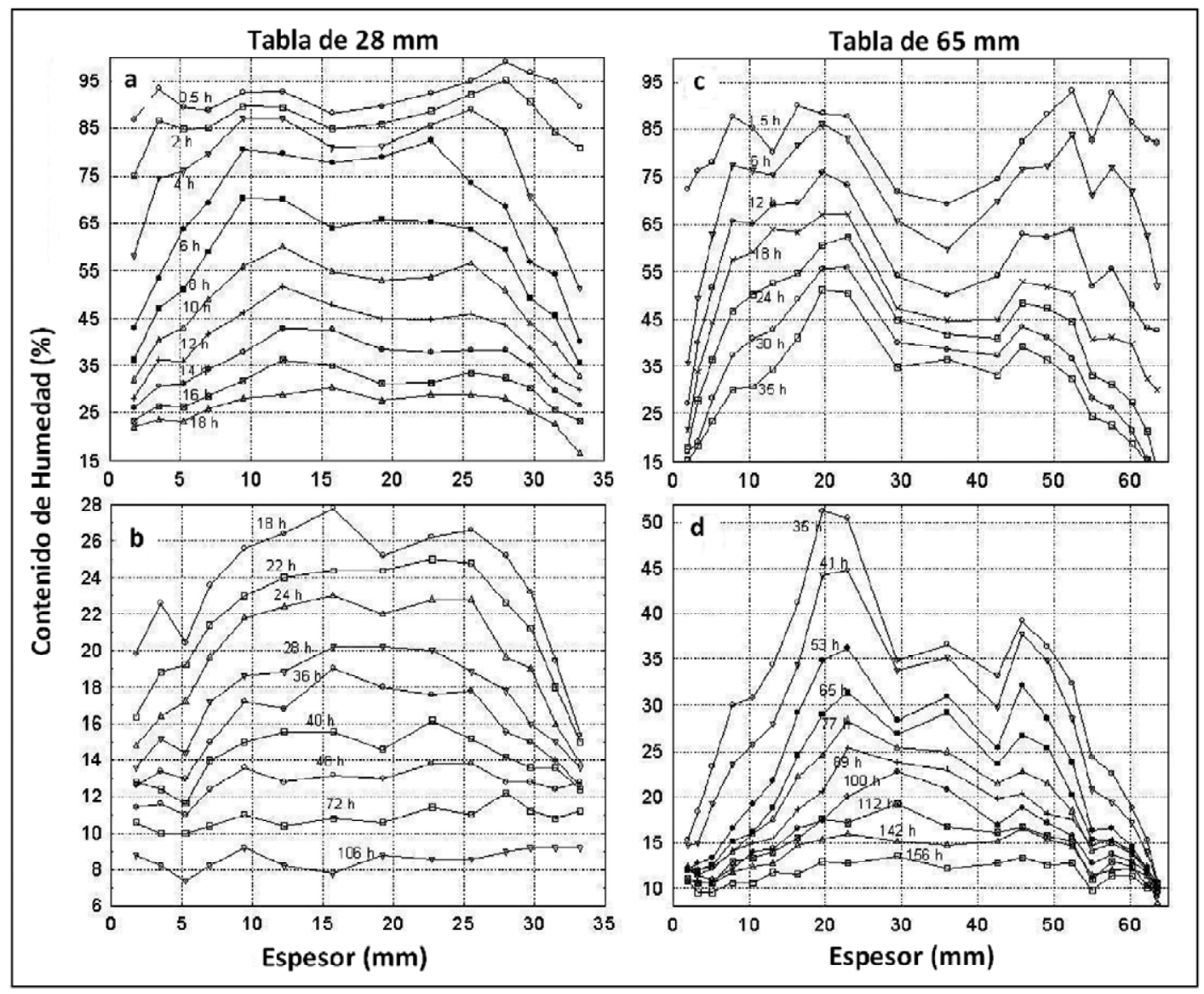

Figura 3. Evolución espacio-temporal del contenido de humedad para las tablas de $28 \mathrm{~mm}$ y $65 \mathrm{~mm}$ de espesor.

La figura 4 detalla el cuociente $\mathrm{R}^{1}$ entre los espectros promedio de dispersión $\left(\theta=10,5^{\circ}\right)$ y de transmisión $\left(I_{d}(E) / I_{t}(E)\right)$ para una muestra de madera en proceso de secado. La intensidad del espectro de dispersión fue corregida tomando en cuenta el tamaño real del elemento sensible del detector dentro del arco de $360^{\circ}$. La muestra inicialmente se encontraba a un contenido de humedad promedio de $188 \%$ y al cabo de $39,2 \mathrm{~h}$ a un contenido de humedad de $80 \%$. Conforme a la pérdida de contenido de humedad se aprecia un descenso sistemático del cuociente. En rigor, ambas intensidades se reducen gradualmente, sin embargo, la intensidad de dispersión lo hace en forma más marcada como consecuencia de la menor cantidad de agua líquida presente en la muestra. Se aprecia que en ausencia de agua, la muestra continúa generando una señal de dispersión de rayos $\mathrm{X}$ no nula. Análisis de la variabilidad angular de esta señal de fondo señalan que esta señal proviene de la fase amorfa de la madera y que su intensidad es proporcional a la densidad de la madera (Baettig y Perré 2006). 


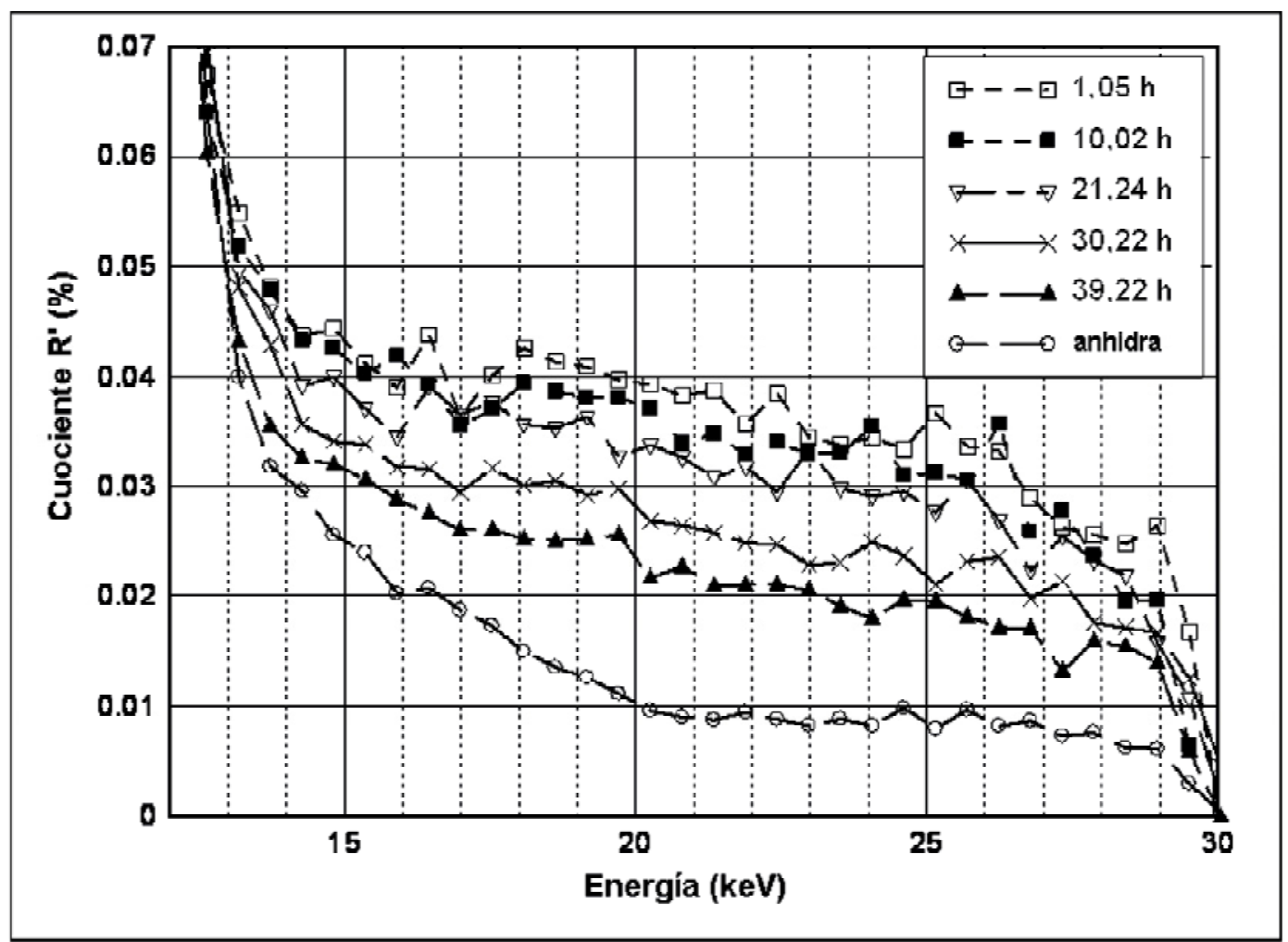

Figura 4. Cuociente $R^{\prime}=I_{d}(E) / I_{t}(E)$ medido para una muestra en proceso de secado. Angulo $2 \theta=10,5^{\circ}$ y tubo con ánodo de cobre alimentado a $30 \mathrm{kV}$. 


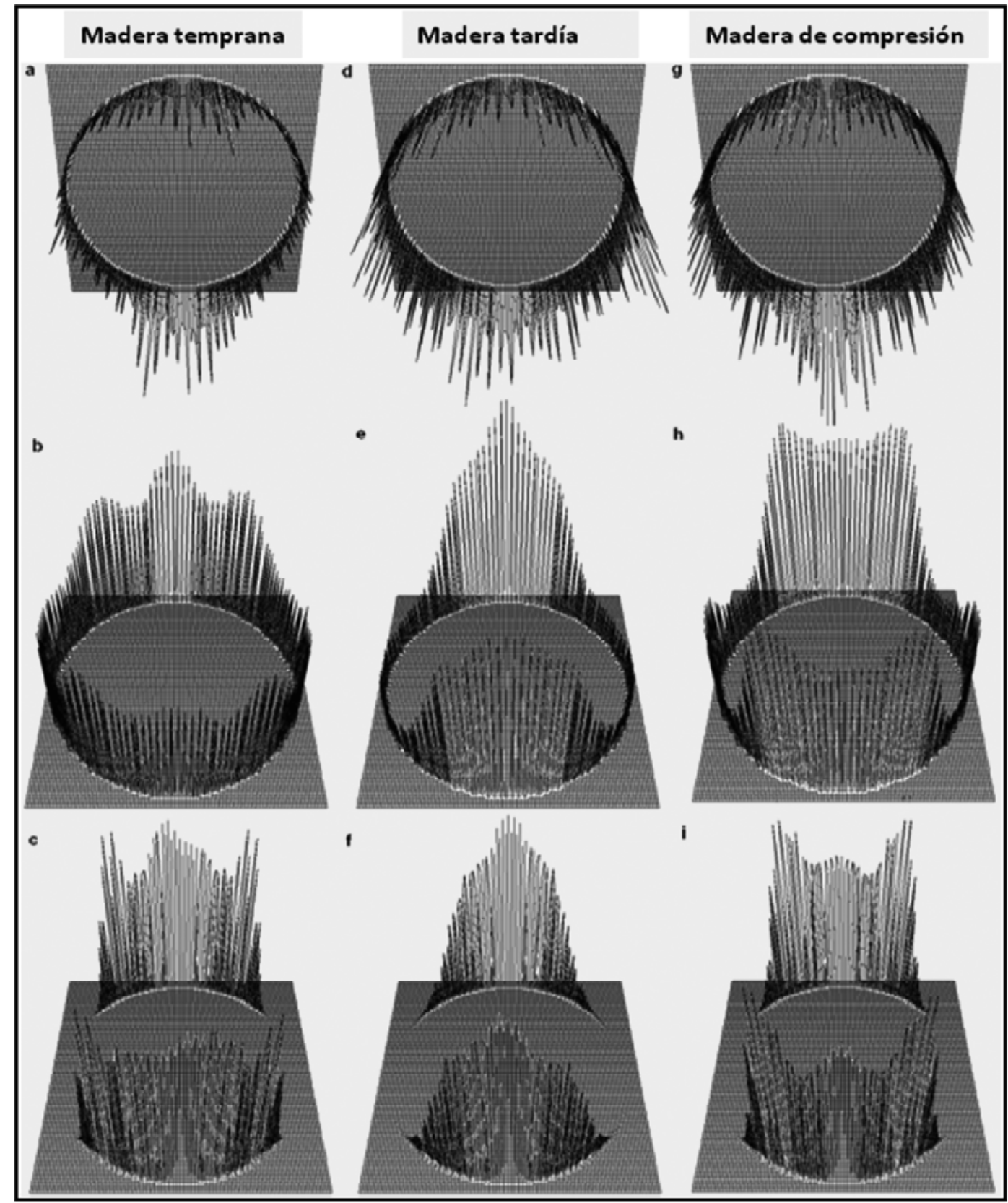

Figura 5. Diagramas polares representando la orientación de la pared celular (a, d, g), el perfil de difracción experimental $(b, e, h)$ y el perfil de difracción teórico (c, f, i) para 3 muestras de madera.

La figura 5 presenta los resultados de experimentos de difracción de rayos $\mathrm{X}$ del plano atómico (002) en muestras de madera temprana, madera tardía y madera de compresión respectivamente. Cabe señalar que en esta figura, el rayo X incidente se aproximaría verticalmente hacia abajo con respecto a los diagramas. Los tres gráficos superiores (Fig. 5 a,d,g) presentan la distribución angular de la pared celular obtenida por tratamiento digital de imágenes microscópicas realizadas por medio de un microscopio electrónico. Los gráficos intermedios (Fig. 5 b,e,h) presentan el perfil de difracción medido 
experimentalmente y seleccionado por energía en los alrededores de la línea $K \alpha M o(17,5 \mathrm{keV})$ para un ángulo $2 \theta=10,5^{\circ}$. Los gráficos inferiores (Fig. 5 c,f,i) presentan el perfil teórico de difracción simulado a partir de la distribución angular de la pared celular para unos valores del ángulo microfibrilar (AMF) elegidos arbitrariamente intentando asimilarse a la forma del perfil de difracción experimental. La altura de las curvas fue ajustada y está expresada en escala relativa.

La Fig. 5a muestra que la pared celular de la madera temprana presenta 2 direcciones principales lo que permite deducir que se encuentra conformada por células de forma aproximadamente rectangulares. En la figura $5 \mathrm{~b}$ se observa que cada peak de intensidad de difracción está formado por tres peaks independientes. Análisis presentados en Baettig (2005) revelan que los dos peaks laterales corresponden a las contribuciones de las paredes laterales con respecto a la dirección del rayo $\mathrm{X}$ incidente. Por su parte, el peak central en realidad está conformado por 2 peaks muy juntos que corresponden a la contribución a la difracción de las partes frontal y posterior de la pared celular con respecto al rayo $\mathrm{X}$ incidente. La figura $5 \mathrm{c}$ es el resultado de la simulación del perfil de difracción calculada a partir de la figura 5a. No se logró obtener una buena correspondencia de forma con la figura 5b. El resultado mostrado fue conseguido usando un $\mathrm{AMF}$ de $37^{\circ}$. Ahora bien, la figura $5 \mathrm{~d}$ obtenida para la muestra revela una distribución de pared celular relativamente homogénea, lo cual revela secciones transversales relativamente circulares. Comparando la figura $5 \mathrm{e}$ con la $5 \mathrm{~b}$, se aprecia que los peaks laterales han prácticamente desaparecido, hecho que revela una reducción del AMF. La mejor correspondencia entre el perfil experimental (Fig. 5e) y el perfil simulado (Fig. 5f) se logró con un AMF de $24^{\circ}$. La figura $5 \mathrm{~g}$ revela una sección transversal de las paredes celulares casi redonda, lo cual, es una de las características más notorias de la madera de compresión. El perfil de difracción está conformado además de los peaks "normales" por otros más pequeños a $90^{\circ}$ y $270^{\circ}$ con respecto al rayo incidente. Estos peaks adicionales han sido explicados por Cave (1997) y Andersson et al. (2000) como provenientes de las microfibrilas de la capa S1 que es de mayor espesor que en madera normal. A su vez, el peak principal presenta una depresión central que también ha sido descrita en madera de compresión por Cave (1997) y Andersson et al. (2000). La mejor correspondencia entre perfil experimental (Fig. 5h) y el perfil simulado (Fig. 5i) se logró usando un AMF igual a $35^{\circ}$.

\section{CONCLUSIONES}

El objetivo de este artículo ha sido el de demostrar la factibilidad técnica de utilizar fuentes policromáticas de rayos $\mathrm{X}$ en experimentos relacionados con la madera en que normalmente se requiere de fuentes de rayos $\mathrm{X}$ monocromáticas. Se presentaron los resultados iniciales de 3 tipos de experimentos, basados en espectrometría de rayos X. Los resultados de estos experimentos revelan la factibilidad técnica de utilizar radiaciones policromáticas, es decir, rayos X provenientes directamente desde tubos sin pasar por monocromatizadores. Esto es posible gracias a la utilización de detectores espectrométricos que permiten seleccionar el o los rangos de energía de interés para determinadas aplicaciones.

En el primer experimento descrito, conformado por un escaneo en espesor durante un secado convectivo, se logró registrar la evolución espacial y temporal del contenido de humedad de la tabla. Los perfiles de humedad obtenidos prueban incontestablemente una pérdida de humedad mucho más lenta en el interior de la madera que en la periferia. Este tipo de registros son extremadamente útiles en la investigación del comportamiento frente al secado de algunas maderas, permitiendo por ejemplo, calcular parámetros como permeabilidad, que son necesarios para alimentar simuladores de cuantitativos de secado tal como TransPore.

En un segundo experimento se demostró la posibilidad de explotar la firma en dispersión de rayos $\mathrm{X}$ generada por las moléculas del agua para estimar el contenido de humedad de de la madera. Se encontró la existencia de una marcada reducción en la intensidad espectral de los rayos X dispersados 
a $10,5^{\circ}$ a medida que la madera iba reduciendo su humedad. Lamentablemente, al estado actual, no ha sido posible calcular el contenido de humedad de las muestras debido a la presencia de una señal de fondo en el espectro, originado por la fase amorfa de la madera.

Finalmente, se ha demostrado la factibilidad de utilizar rayos X no monocromáticos para cuantificar la intensidad de difracción de rayos X del plano atómico (002) de la celulosa en la madera. El escaneo angular de esta intensidad permitió obtener unos perfiles de difracción que pudieron ser confrontados a perfiles obtenidos por medio de un modelo geométrico. Si bien los resultados obtenidos deben ser tomados con precaución, se cree haber encontrado una alternativa promisoria para la estimación del ángulo microfibrilar de la madera, usando un dispositivo de relativa simplicidad.

\section{REFERENCIAS}

Andersson, S.; Serimaa, R.; Torkkelo, M.; Paakkari, T.; Saranpaa, E. 2000. Microfibril angle of Norway spruce (Picea abies (L.) Karst) compression wood: comparison of measuring techniques. Journal of Wood Science 46: 343-349.

Baettig, R. 2005. Exploitation d'un rayonnement X poly-energétique pour la détermination de la teneur en eau et de l'angle de microfibrilles du bois : approche théorique et expérimentale. Thèse ENGREF. Nancy. France.

Baettig R, Rémond R, Perré P. 2006. Measuring moisture content profiles in a board during drying: a polychromatic X-ray system interfaced with a vacuum/pressure laboratory kiln. Wood Science and Technology 40(4):261-274.

Baettig, R.; Perré, P. 2006. Uso de dispersión coherente de rayos X para la determinación simultánea del contenido de humedad y densidad de la madera: estudio exploratorio. (En preparación).

Bauters, T.; DiCarlo, D.; Steenhuis, T. 2000. Rapid, quantitative tracking of fluid flow in soils with synchrotron x-rays. Chess Newslettere.54-58. CHESS - Cornell University.

Bentz, D.; Hansen, K. 2000. Preliminary observations of water movement in cement pastes during curing using x-ray absortion. Cement and concrete research 30(7):1157-1168.

Cave, I. 1997. Theory of X-ray measurement of microfibril angle in wood. Part 2: The diffraction diagram X-ray diffraction by materials with fibre type symmetry. Wood Science and Technology 31(4): 225-234.

Clarijs, M.; Bom, V.; Kolar, Z.; van Eijk, C.; Frieling, J.; Cheers, A.; Reimerink, A. 1999. Optimized x-ray spectra for multiphase-flow measurements. Proceeding of Computerized Tomography for industrial applications and image proccesing in radiology. March 15-17. 1999. DGZfP - German Society for Non-Destructive Testing. Berlin. Germany.153-157

Dinten, J.; Robert-Coutant, C.; Darboux, M. 2001. Dual-energy x-ray absortiometry using a 2D digital radiography detector. Application to bone densitometry. Proceedings of SPIE. Ed. Larry E. Antonuk, Martin J. Yaffe. Volume 4320. Medical Imaging 2001: Physics of Medical Imaging, San Diego, CA, USA. 18 February 2001. pp. 459-468.

Evans, R.; Stuart ,S.; Van der Touw, J. 1996. Microfibril angle scanning of increment cores by xray diffractometry. Appita Journal 49(6): 411-414. 
Evans, R. 1999. A variance approach to the x-ray diffractometric estimation of microfibril angle in wood. Appita Journal 52(4): 283-294.

Hansen, K.; Genward, L.; Singh, K. 1999. Dual-energy absortiometry for the simultaneous determination of density and moisture content in porous structural materials. Proceeding of the 5th symposium on Building Physics - Gotenburg.

Hu, J.; Stroeven, P. 2003. X-ray absorption study of drying cement paste and mortar. Cement and Concrete Research 33: 397-403.

Johns, P.; Wismayer, M.; Leclair, R. 2002. The need for cross section data for medical x-ray scatter imaging. 5th Annual canadian light source users meeting, Saskatoon. Canada.

Key, M. 1999. Gas microestructure x-ray detectors and tomographic multiphase flow measurement. School of Physical Sciences. University of Surrey. Surrey.

Kretschmann, D.; Alden, H.; Verrill, S. 1997. Variations of microfibril angle in loblolly pine: comparison of iodine crystallization and x-ray diffraction techniques. Microfibril angle in wood. B. G. Butterfield. Westport, New Zealand: 157-176.

Laskey, M.; Phil, D. 1996. Dual-energy X-ray absorptiometry and body composition. Nutrition (12): 45-51.

Létang, J.; Freud, N.; Peix, G. 2003. Optimal calibration via virtual x-ray imaging for dualenergy techniques: application to glass wool. Proceedings of SPIE - Sixth International Conference on Quality Control by Artificial Vision.

Magalhaes, S.; Eichler, J.; Goncalves, O. 1995. Calculation on X-ray scattering of $17.4 \mathrm{keV}$ radiation and image degradation in mammography. Nuclear Instruments and Methods in Physics Research B. 95:87-90.

McBride, J.; Miller, C. 1994. Nondestructive measurements of phase fractions in multiphse porousmedia experiments by using x-ray attenuation. Center for multiphase research news University of North Carolina. 1: 1-5.

Norel, G. 1965. Etude des milieux poreux par absorption d'un rayonnement X ou gamma émis par une source radioactive. Faculté des Sciences. Paris, Université de Paris: 116 p.

Oostrom, M.; Dave, JH. 1990. Calibration and automation of a dual-energy gamma system for applications in soil science. Agronomy and soils departamental series report N-145 Auburn University - Auburn.

Perré, P.; Thiercelin, F. 1997. Determination de profils d'humidite dans le bois par détection directe de rayons-X: validation de la méthode. Les cahiers scientifiques du bois. Volume 1. Instrumentation. Nancy, ARBOLOR.

Perré, P. ; Thiercelin, F. 2004. Evidence of dual scale porous mechanism during fluid migration in hardwood species. (I) Using the attenuation of a polychromatic X-ray beam to determine the evolution of moisture content during imbibition of beech. Chinese Journal of Chemical Engineering 12(6): 773 782. 
Perré, P.; Turner, I. 1996. Using a set of macroscopic equations to simulate heat and mass transfer in porous media: some possibilities illustrated by a wide range of configurations that emphasize the role of internal pressure, In: Numerical methods and mathematical modelling of the drying process, Eds. Turner, I., Mujumdar, A., Marcel Dekker, 83-156.

Perré, P.; Turner, I. 1999. A 3D version of TransPore: A Comprehensive Heat and Mass Transfer Computational Model for Simulating the Drying of Porous Media Int. J. Heat Mass Transfer 42: 45014521.

Poletti, ME.; Gonçalves, OD.; Mazzaro, I. 2004. Measurements of X-ray scatter signatures for some tissue-equivalent materials. Nuclear Instruments and Methods in Physics Research B 213: 595598.

Schena, G.; Chiaruttini, C.; Dreossi, D.; Olivo, A.; Pani, S. 2002. Grade of fine composite mineral particles by dual-energy X-ray radiography. International Journal of Mineral Processing 67:101122.

Tartari, A.; Taibi, A.; Bonifazzi, C.; Baraldi, C. 2002. Updating of form factor tabulations for coherent scattering of photons in tissues. Physics in Medicine and Biology 47: 163-175.

Verrill, S.; Kretschmann, D.; Herian, V. 2001. JMFA - A graphically interactive java program that fits microfibril angle x-ray diffraction data. USDA Forest Service Forest Products Laboratory Research Note \#283. Madison, Wisconsin. 
